\title{
Epigenetic aspects of telomeric chromatin in Arabidopsis thaliana
}

\author{
Won Kyung Lee \& Myeon Haeng Cho ${ }^{*}$ \\ Department of Systems Biology, Yonsei University, Seoul 03722, Korea
}

Telomeres are nucleoprotein complexes at the physical ends of linear eukaryotic chromosomes. They protect the chromosome ends from various external attacks to avoid the loss of genetic information. Telomeres are maintained by cellular activities associated with telomerase and telomerebinding proteins. In addition, epigenetic regulators have pivotal roles in controlling the chromatin state at telomeres and subtelomeric regions, contributing to the maintenance of chromosomal homeostasis in yeast, animals, and plants. Here, we review the recent findings on chromatin modifications possibly associated with the dynamic states of telomeres in Arabidopsis thaliana. [BMB Reports 2019; 52(3): 175-180]

\section{INTRODUCTION}

In eukaryotic cells, the chromosome ends are protected by telomeres from inappropriate fusion and degradation, and incomplete DNA synthesis during DNA replication (1). Maintenance of the proper structure and function of telomeres is essential for the conservation of genetic information, chromosomal stability, and thus, cell survival (2). Eukaryotic chromosome ends are mainly divided into telomeres and adjacent subtelomeric regions (3). Telomeres consist of double-stranded repetitive G-rich DNA with single-stranded overhangs. When deletion of DNA sequences at the end of chromosomes is caused by various cellular events, telomerase accesses and adds telomeric repeats to critically short telomeres preferentially with its reverse transcriptase activity using its own internal RNA template, thereby effectively stabilizing telomere length (4). Telomere-binding proteins in mammals, known as shelterin, participate in the formation and maintenance of the specialized telomeric structure (T-loop) and the precise regulation of telomere length (5). Moreover,

*Corresponding author. Tel: +82-2-2123-4460; Fax: +82-2-3125657; E-mail: mhcho@yonsei.ac.kr

https://doi.org/10.5483/BMBRep.2019.52.3.047

Received 10 January 2019

Keywords: Arabidopsis thaliana, DNA methylation, Histone deacetylation, Histone methylation, Telomeres they interact with several non-telomere-binding proteins involved in DNA repair and recombination, contributing to the integrity and dynamics of the telomeres (6). Subtelomeric regions in humans are composed of degenerated telomeric repeat sequences with a high density of methylated CpG DNA sequences (7). The heterochromatic nature of subtelomeric regions influences the epigenetic silencing of telomereadjacent genes by the telomere position effect (TPE) and abnormal chromosome recombination in yeast and humans (8). Non-coding RNAs containing telomeric repeats (TERRA) are generated in these regions (9). TERRA expression is regulated by the chromatin state, and in turn, telomere length is regulated by the expression level of TERRA $(9,10)$. Unique structures of telomeres and characteristics of telomere-related proteins have been observed in yeast, animals, and plants, demonstrating that these are evolutionarily-conserved and essential features of telomeres $(5,11,12)$.

Telomeres, like centromeres, are generally defined as heterochromatic regions of the genome, characterized by increased chromatin condensation and decreased access to regulatory proteins (13). Many researchers have tried to understand what kinds of epigenetic marks are enriched in telomeric chromatin, how telomeres are regulated by these epigenetic modifications at the level of chromatin state, and thus, how these telomeric modifications affect their biological function. A repressive chromatin environment, formed by histone modifications and DNA methylation at telomeres and subtelomeric regions, has been shown to control the telomeric structure and function in yeast and mammals $(14,15)$.

In yeast, $\mathrm{NAD}^{+}$-dependent histone deacetylase Sir2 is a component of the silent information regulator (SIR) complex, which is implicated in the silencing of subtelomeric chromatin (16). Sir2 is recruited to telomeres by Rap1, and its HDAC (histone deacetylase) activity is necessary for its proper localization on telomeres and regulation of the heterochromatin structure in telomeres. Mammalian SIRT6 is specifically associated with telomeric chromatin and the loss of its HDAC activity results in the hyperacetylation of telomeric histone $\mathrm{H} 3 \mathrm{~K} 9$, telomere dysfunction, premature cellular senescence, and impaired silencing of telomere-proximal genes, indicating that SIRT6 modulates the telomeric chromatin structure (17, 18). Mammalian TPE is HDAC- and telomere lengthdependent $(19,20)$. 
In addition, histone methyltransferases involved in the trimethylation of $\mathrm{H} 3 \mathrm{~K} 9$ and $\mathrm{H} 4 \mathrm{~K} 20$, which are the main histone marks of telomeric and subtelomeric heterochromatin, contribute to the regulation of telomere length in mammals $(21,22)$. Moreover, heavily methylated DNA at mammalian subtelomeric regions are associated with the regulation of telomere elongation, TERRA expression, and stability $(23,24)$.

These studies present strong evidence that epigenetic modifications are involved in the composition of telomeric chromatin and have important roles in its regulation. Despite these outstanding achievements, the precise composition of epigenetic marks at telomeric chromatin and the relationship between telomeres, telomere-binding proteins, and these epigenetic regulators are not fully understood yet. Especially, many chromatin modifying proteins associated with epigenetic modifications in various target loci do not contain DNA-binding domains, thus prompting questions regarding how these proteins find their target loci.

Recent data in Arabidopsis provide convincing evidence to support the role of telomere-binding proteins in epigenetic events which regulate telomeres. In this review, we provide a brief overview, with a special emphasis on the epigenetic regulation of telomeric chromatin in Arabidopsis.

\section{FUNCTION OF EPIGENETIC REGULATORS AND REGULATORY FACTORS OF TELOMERIC CHROMATIN IN ARABIDOPSIS}

\section{Histone modifications}

In eukaryotes, the combination of different post-translational modifications (methylation, acetylation, phosphorylation, ubiquitination, sumoylation, and ADP-ribosylation) on the $\mathrm{N}$-terminal tails of histone dictates the rapid change of the chromatin state into a transcriptionally-active euchromatin or silent heterochromatin state (25). Histone acetylation and deacetylation of lysine residues are reversible processes, mediated by histone acetyltransferases (HATs) and histone deacetylases (HDACs), respectively (26).

Based on the sequence homology to yeast HDACs, plant HDACs are classified into three major families: RPD3/HDA1, SIR2, and HD2 (27). Studies have reported that several histone modifiers, as partners of telomere-binding proteins, are required for regulation of the chromatin state of Arabidopsis telomeres. SNL1, one of the SIN3 orthologues identified in Arabidopsis, was shown to interact with telomere-binding proteins, AtTBP1 and AtTRP2, by yeast two-hybrid screening, suggesting potential functions for SNL1 in telomere maintenance (28). In addition, two different types of HDACs, HDT4, and HDA6, directly interacted with AtTRB2, a telobox-containing telomere-binding protein, and played a role in the maintenance of telomere length in Arabidopsis (29). HDT4, a plant-specific HD2-type HDAC, is a putative $\mathrm{H} 3$ lysine 27 deacetylase. HDA6, an RPD3/HDA1-type HDAC, deacetylates the acetylated lysine residues at $\mathrm{H} 3$ and $\mathrm{H} 4$.
HDA6 is known to function in rDNA silencing and in the responses to various abiotic stresses $(30,31)$. Recently, our study showed that Sir2-type AtSRT1 was also associated with telomeric chromatin through the direct interaction with AtTRB2 and that AtSRT1 was characterized as a putative $\mathrm{NAD}^{+}$-dependent $\mathrm{H} 3 \mathrm{~K} 9$ deacetylase in Arabidopsis (unpublished data).

Through Southern blot analysis using mutants, it was shown that Arabidopsis HDACs negatively regulated the telomere elongation, similar to AtTRB2 (29). In addition, telomeric-ChIP (T-ChIP) data in these studies showed that telomere-binding proteins carried out several essential tasks, such as a recruitment of epigenetic regulators to telomeres, as well as the binding to telomeric repeats in Arabidopsis (29). It is assumed that the epigenetic regulators will be precisely located in telomeres through the interaction with telomere-binding proteins, and a change in the chromatin state from heterochromatin to euchromatin at telomeres by mutation of the HDACs will permit the appropriate conditions for further access of molecules, such as telomerase, to affect telomere elongation.

These reports also suggest the possible regulation of histone modification via combinatorial composition and competition between the different kinds of HDACs. This implies that the coordination between the various HDACs at telomeres is a universal regulatory mechanism in yeast and plants (32). In budding yeast, reports have shown that different types of HDAC proteins competed with each other for appropriate adjustment of the boundary element on telomeric chromatin. Rpd3 HDAC protein is necessary to restrict the SIR complex to telomeres and thus, modulates a barrier to prevent the spread of the SIR-dependent telomere position effect (32-34). Additionally, the competition of Sir2 HDAC and Sas2 HAT creates flexible boundaries at telomeres in yeast $(35,36)$.

Moreover, it has been noted that histone modifications at lysine 9 and lysine 27 on histone $\mathrm{H} 3$ were significant components of the telomeric chromatin in Arabidopsis. Several observations support the involvement of histone methyltransferases associated with these residues in the regulation of Arabidopsis telomeres. T-ChIP data showed that the H3K9-specific histone methyltransferase KYP was associated with telomeric $\mathrm{H} 3 \mathrm{~K} 9 \mathrm{me}^{2}(37,38)$. In addition, recent findings revealed that telobox-related motifs recruited PRC2 through the interaction between AtTRBs and CLF/SWN, proposing a mechanism essential for $\mathrm{H} 3 \mathrm{~K} 27 \mathrm{me}^{3}$ deposition at a subset of target genes (39). These results strongly confirm the significance of the modifications at $\mathrm{H} 3 \mathrm{~K} 9$ and $\mathrm{H} 3 \mathrm{~K} 27$ in telomeres and the functions of telomere-binding proteins with their sequence-specific DNA-binding activity in the targeting of telomere-associated proteins to telomeres.

\section{DNA methylation}

DNA methylation is conserved in many eukaryotic organisms. Once established, DNA methylation is inherited through 
mitosis, and often through meiosis, and this provides an effective epigenetic mark (40). In Arabidopsis, MET1, homologous to mammalian Dnmt1, is mainly involved in CpG DNA methylation. Chromomethyltransferase CMT3 is unique to plants and important for $\mathrm{CpNpG}$ and asymmetric methylation (41).

The chromatin state is regulated by interplay between the epigenetic modifications in eukaryotes (42). In some cases, the modifications on histone tails provide the binding site for effector proteins. It has been proposed that the interconnections between the epigenetic modifications act as signals to each other for establishing and maintaining stable epigenetic states. In Arabidopsis, the methylation of $\mathrm{H} 3 \mathrm{~K} 9$ controls DNA methylation by CMT3 because CMT3 recognizes the methylated lysines 9 and 27 of histone $\mathrm{H} 3$ selectively, and further catalyzes the methylation at $\mathrm{CpNpG}$ sequences (43). Additionally, the direct interaction between epigenetic regulators results in effective regulation of the chromatin state. HDA6 mediates the silencing of the heterochromatic regions by physical interaction with MET1 $(44,45)$.

In Arabidopsis, the results from Southern blot analysis and Chop-PCR using McrBC endonuclease indicated that the acting loci of CMT3 were separated from those of MET1; CMT3 and MET1 mainly regulated DNA methylation in the $4 R$ subtelomeric region and in 300-bp interstitial telomeric sequences (ITSs), respectively, and that they negatively regulated telomere length (29). Consistent with previous studies $(38,46)$, this result confirmed that non-CpG DNA methylation comprised the majority of DNA methylation in telomeric repeat sequences in Arabidopsis, demonstrating an essential role for CMT3 in the maintenance of non-CpG DNA methylation in telomeres/subtelomeres. Southern blot analysis using restriction enzymes also showed a similar pattern of DNA methylation in telomeric repeat sequences between hda6 and $\mathrm{cmt} 3$, but not the met 1 mutant, suggesting that HDA6 contributes to non-CpG DNA methylation by collaboration with CMT3 in telomeres/subtelomeres.

In contrast to the effect of deacetylated H3K9 on telomeric DNA methylation, an hdt4-1 mutant showed no alteration in DNA methylation of telomeric chromatin (29). It is presumed that H3K27 modifications regulate chromatin by separate pathways (47). In fact, it has been reported that defects in the H3K27 monomethyltransferases in Arabidopsis resulted in chromatin decondensation by causing the over-replication of heterochromatin without any effects on DNA methylation (48-50). Thus, the lack of alteration in DNA methylation on telomeric chromatin in the hdt4-1 mutant was possibly due to the different effects of these two histone modifications on DNA methylation.

In addition to DNA methyltransferases and histone modifiers, chromatin structural proteins also control the composition and level of epigenetic marks. Chromatin remodeling factor DDM1, a SWI2/SNF2 orthologue, has been previously reported to facilitate heterochromatin formation by promoting the access of DNA methyltransferase to the heterochromatin $(51,52)$. Southern blot analysis using a $d d m 1$ mutant indicated that DDM1 controlled subtelomeric CpG methylation, but not $\mathrm{H} 3 \mathrm{~K} 9 \mathrm{me}^{2}$ or non-CpG methylation at subtelomeric regions, contributing to the formation of subtelomeric heterochromatin in Arabidopsis (38). In contrast, DDM1 affected the level of $\mathrm{H}_{3} \mathrm{~K} 9 \mathrm{me}^{2}$ and $5-\mathrm{mC}$ at the Ta3 retrotransposon (38). These results note that heterochromatin formation at subtelomeric regions is distinguished from that at other heterochromatic loci.

Most recent study on DDM1 reported that telomere shortening in a late generation $d d m 1-2$ mutants was not related to telomerase activity or TERRA expression (53). Instead, telomere shortening in a ddm1-2 mutant was seemed to be a by-product of the increased recombination caused by the hypomethylation of DNA, based on the notion that there is correlation between telomere length, telomere recombination, and transposon activation. However, it is still unclear whether DDM1 directly influences the regulation of the telomeric chromatin state. Further comprehensive studies should be conducted to determine the regulatory pathway of telomeric-/ subtelomeric chromatin involving chromatin remodeling factors, such as DDM1.

\section{Epigenetic state of telomere chromatin}

Heterochromatin is stably-inherited and thus, must contain one or more epigenetic marks to direct its maintenance during cell division (54). Heterochromatin is generally characterized by $\mathrm{H} 3 \mathrm{~K} 9 \mathrm{me}^{1,2}, \mathrm{H} 3 \mathrm{~K} 27 \mathrm{me}^{1,2}, \mathrm{H} 4 \mathrm{~K} 20 \mathrm{me}^{1}$, and methyl cytosine, whereas euchromatin is characterized by $\mathrm{H} 3 \mathrm{~K} 4 \mathrm{me}^{1,2,3}$, $\mathrm{H} 3 \mathrm{~K} 36 \mathrm{me}^{1,2,3}$, H4K20me $\mathrm{e}^{2,3}$, and histone acetylation in Arabidopsis thaliana (55). The chromatin state of certain loci is determined by the quantitative and qualitative composition of different epigenetic modifications.

In mammals, telomeric nucleosomes have a more compact structure with heterochromatic features (15). There is a higher density of $\mathrm{H} 3 \mathrm{~K} 9 \mathrm{me}^{3}, \mathrm{H} 4 \mathrm{~K} 20 \mathrm{me}^{3}$, and $\mathrm{HP} 1$ (heterochromatin protein 1) in mammalian telomeres and subtelomeric regions, as well as higher levels of methyl cytosine by DNMT1 and DNMT3a/b in subtelomeric regions. Heterochromatic marks at telomeres have been proposed to act as negative regulators of telomere elongation. Interestingly, loss of heterochromatic marks at telomeres did not seem to affect TRF1 and TRF2 binding, indicating that shelterin recruitment was uncoupled from telomeric chromatin regulation $(21,22,56,57)$. Most recently, a study on the epigenetic characteristics of human telomeres revealed that telomeres had lower levels of $\mathrm{H} 3 \mathrm{~K} 9 \mathrm{me}^{3}$ and enriched levels of H4K2Ome ${ }^{1}$ and H3K27Ac marks compared to certain heterochromatic loci in different human cell lines (58). In addition, several cancer cell lines that maintain their telomeres through ALT exhibited heterochromatic levels of $\mathrm{H} 3 \mathrm{~K} 9 \mathrm{me}^{3}$. This suggested that telomeres in ALT cells became 'subtelomeric' according to their heterogeneous length and sequence composition containing degenerated 
telomeric repeats via recombination with subtelomeric regions $(59,60)$. It also implies that the mechanism of telomere maintenance by recombination in ALT is considerably different from that in canonical conditions whereby telomeres are elongated by telomerase. These results highlight the differences in several previous reports. It appears that the effect of the epigenetic features of telomeres and subtelomeric regions on their functions in humans is still an open question.

Analysis of epigenetic marks on the chromatin structure of Arabidopsis telomeres revealed that telomeres were not heterochromatic, whereas subtelomeric regions and ITSs exhibited heterochromatic features in Arabidopsis (38, 46, 61-63). Methyl cytosine, H3K9me ${ }^{2}$, and $\mathrm{H} 3 \mathrm{~K} 27 \mathrm{me}^{1}$ were observed in adjacent subtelomeric regions and ITSs, while $\mathrm{H} 3 \mathrm{~K} 27 \mathrm{me}^{3}$, known as a repressive mark in euchromatin genes, was found in telomeres. The heterochromatic state of subtelomeric regions is formed by HDACs, histone methyltransferases, DNA methyltransferases, chromatin remodeling protein, and molecules involved in the RdDM pathway $(29,38,46)$. Separate studies showed a consistent pattern of distribution on methyl cytosine, $\mathrm{H} 3 \mathrm{~K} 9 \mathrm{me}^{2}$, and TERRAARRET up to $2 \mathrm{~kb}$ from the chromosome ends in Arabidopsis, although their distribution patterns differed in each chromosome arm (http://epigenomics.mcdb.ucla.edu/ DNAmeth/). The correlations between epigenetic events mentioned above again suggest that the RdDM pathway, histone modification, and DNA methylation processes may be involved in common events in telomeric heterochromatin formation. It has been noted that Arabidopsis RdDM mutants had no alterations in telomere length compared to the wild-type, in contrast to the mutants of epigenetic regulators, including HDAC proteins and DNA methyltransferases (29, 46). Based on these findings, it is inferred that the mechanism of telomere length regulation mediated by histone modifiers and DNA methyltransferases is distinct from that of the RdDM pathway. It is also assumed that the heterochromatin state at subtelomeric regions in Arabidopsis is important to the formation and maintenance of the boundary element between telomeres and its distal euchromatin genes by buffering the propagation of TPE, similar to that in yeast and mammals.

Although the epigenetic characteristics of Arabidopsis telomeres is impressive, it is arguable whether Arabidopsis telomeres are euchromatic because of the technical limitation of hybridization-based sequencing technology. It is not possible to directly compare the quality and quantity of epigenetic marks side-by-side among telomeres, subtelomeric regions, and ITSs using this method. In the most recent report by the same research group, an improved procedure based on statistical analysis of multiple ChIP-seq experiments was performed to exclude the interferences of subtelomeric regions and ITSs (58). Nevertheless, to define the chromatin state of telomeres composed of only TTTAGGG repeats, it is necessary to make up the advanced experimental techniques to finely separate telomeric repeat sequences-harboring regions, such as
ITSs, degenerated repeat sequences in subtelomeric regions, and telomeric repeats in telomeres. Moreover, the epigenetic characteristics of mammals and Arabidopsis are still controversial. Results from the hybridization-based sequencing method suggested that Arabidopsis telomeres were less heterochromatic than subtelomeric regions or ITS. However, 'less heterochromatic' does not mean 'euchromatic'. Therefore, it is safe to say that these results showed a mix of both euchromatic and heterochromatic marks at plant telomeres, as described by Galati et al. (15).

\section{PERSPECTIVES}

The principal functions of the heterochromatic state of telomeres are the protection of chromosome ends, the regulation of telomere length, and the suppression of recombination events at the telomeres. Recent findings noted that DNA methylation and histone modifications were involved in the regulation of chromatin status and the elongation of telomeres in many species, and suggested the possibility that cooperation and/or competition of these epigenetic modifications are required for the subtle and elaborate regulation of telomeric and subtelomeric chromatin state, thus maintaining the homeostasis of chromosomes.

However, the biological meaning of the formation of chromosome ends and the regulation of the chromatin state at the chromosome ends is considerably unrevealed and disputable. Especially, there are many unidentified pieces in the puzzle of the epigenetic regulation of Arabidopsis telomeres. Therefore, it is necessary to find the role of various molecules which affect telomeric chromatin state, for instance, chromatin-remodeling factors, histone chaperones, and smallRNA related molecules.

Moreover, the unrevealed functions of telomere-binding proteins are still remained. In humans, hTRF1 and hTRF2 are associated with ITSs, as well as telomeres, contributing to the stability of chromosomes. Arabidopsis ITSs are located in subtelomeric regions and pericentromeres. ITSs are known to be hot-spots for chromosomal recombination. Genetic and epigenetic regulation of ITSs is essential to stabilize the ITSs. In addition, hTRF2 is involved in the nucleosomal organization of heterochromatic marks in telomeres and facilitates the heterochromatin formation through associations with TERRA RNAs and other heterochromatin factors, such as HP1 (56, 64, 65). Similarly, AtTRB2 displays binding activity to telomeric repeat sequences, several epigenetic regulators, and histone $\mathrm{H} 3$. Moreover, considering the unique binding activity of AtTRB2 both to telomeric- and degenerated repeat sequences (66), it has been suggested that epigenetic regulators recruited by DNA-bound AtTRB2 target and function at telomeres, as well as at subtelomeric regions and ITS. Therefore, the relevance of telomere-binding proteins, epigenetic regulation, and chromosomal stability should be confirmed and experimental approaches should be designed to discover the 
as yet unknown functions of telomere-binding proteins.

\section{ACKNOWLEDGEMENTS}

This work was supported by the Basic Science Research Program from the Korean National Research Foundation (Grant no. NRF-2016R1D1A1A09919983 to M.H.C.), and in part by the Brain Korea 21(BK21) PLUS program.

\section{CONFLICTS OF INTEREST}

The authors have no conflicting interests.

\section{REFERENCES}

1. O'Sullivan RJ and Karlseder J (2010) Telomeres: protecting chromosomes against genome instability. Nat Rev Mol Cell Biol 11, 171-181

2. Blackburn EH (2010) Telomeres and telomerase: the means to the end (Nobel lecture). Angew Chem Int Ed Engl 49, 7405-7421

3. Louis EJ and Vershinin AV (2005) Chromosome ends: different sequences may provide conserved functions. Bioessays 27, 685-697

4. Gallardo F and Chartrand P (2008) Telomerase biogenesis. RNA Biol 5, 212-215

5. de Lange T (2005) Shelterin: the protein complex that shapes and safeguards human telomeres. Genes Dev 19, 2100-2110

6. Webb CJ, Wu Y and Zakian VA (2013) DNA repair at telomeres: keeping the ends intact. Cold Spring Harb Perspect Biol 5, a012666

7. Brock GJ, Charlton J and Bird A (1999) Densely methylated sequences that are preferentially localized at telomere-proximal regions of human chromosomes. Gene 240, 269-277

8. Ottaviani A, Gilson E and Magdinier F (2008) Telomeric position effect: From the yeast paradigm to human pathologies? Biochimie 90, 93-107

9. Azzalin CM, Reichenbach P, Khoriauli L, Giulotto E and Lingner J (2007) Telomeric repeat containing RNA and RNA surveillance factors at mammalian chromosome ends. Science 318, 798-801

10. Balk B, Maicher A, Dees M et al (2013) Telomeric RNA-DNA hybrids affect telomere-length dynamics and senescence. Nat Struct Mol Biol 20, 1199-1205

11. Zellinger B and Riha K (2007) Composition of plant telomeres. Biochim Biophys Acta 1769, 399-409

12. Kupiec M (2014) Biology of telomeres: lessons from budding yeast. FEMS Microbiol Rev 38, 144-171

13. Lamb JC, Yu W, Han F and Birchler JA (2007) Plant chromosomes from end to end: telomeres, heterochromatin and centromeres. Curr Opin Plant Biol 10, 116-122

14. Blasco MA (2007) The epigenetic regulation of mammalian telomeres. Nat Rev Genet 8, 299-309

15. Galati A, Micheli E and Cacchione S (2013) Chromatin structure in telomere dynamics. Front Oncol 3, 46
16. Jing $\mathrm{H}$ and Lin $\mathrm{H}$ (2015) Sirtuins in epigenetic regulation. Chem Rev 115, 2350-2375

17. Tennen RI, Bua DJ, Wright WE and Chua KF (2011) SIRT6 is required for maintenance of telomere position effect in human cells. Nat Commun 2, 433

18. Michishita E, McCord RA, Berber E et al (2008) SIRT6 is a histone $\mathrm{H} 3$ lysine 9 deacetylase that modulates telomeric chromatin. Nature 452, 492-496

19. Baur JA, Zou Y, Shay JW and Wright WE (2001) Telomere position effect in human cells. Science 292, 2075-2077

20. Koering CE, Pollice A, Zibella MP et al (2002) Human telomeric position effect is determined by chromosomal context and telomeric chromatin integrity. EMBO Rep 3, 1055-1061

21. García-Cao $M, O^{\prime}$ Sullivan $R$, Peters $A H$, Jenuwein $T$ and Blasco MA (2004) Epigenetic regulation of telomere length in mammalian cells by the Suv39h1 and Suv39h2 histone methyltransferases. Nat Genet 36, 94-99

22. Benetti R, Gonzalo S, Jaco I et al (2007) Suv4-20h deficiency results in telomere elongation and derepression of telomere recombination. J Cell Biol 178, 925-936

23. Gonzalo S, Jaco I, Fraga MF et al (2006) DNA methyltransferases control telomere length and telomere recombination in mammalian cells. Nat Cell Biol 8 , 416-424

24. Yehezkel S, Segev Y, Viegas-Péquignot E, Skorecki $K$ and Selig S (2008) Hypomethylation of subtelomeric regions in ICF syndrome is associated with abnormally short telomeres and enhanced transcription from telomeric regions. Hum Mol Genet 17, 2776-2789

25. Jenuwein $T$ and Allis CD (2001) Translating the histone code. Science 293, 1074-1080

26. Gong F and Miller KM (2013) Mammalian DNA repair: HATs and HDACs make their mark through histone acetylation. Mutat Res 750, 23-30

27. Ma X, Lv S, Zhang C and Yang C (2013) Histone deacetylases and their functions in plants. Plant Cell Rep 32, 465-478

28. Bowen AJ, Gonzalez D, Mullins JG, Bhatt AM, Martinez A and Conlan RS (2010) PAH-Domain-Specific Interactions of the Arabidopsis Transcription Coregulator SIN3-LIKE1 (SNL1) with Telomere-Binding Protein 1 and ALWAYS EARLY2 Myb-DNA Binding Factors. J Mol Biol 395, 937-949

29. Lee WK and Cho MH (2016) Telomere-binding protein regulates the chromosome ends through the interaction with histone deacetylases in Arabidopsis thaliana. Nucleic Acids Res 44, 4610-4624

30. Aufsatz W, Stoiber T, Rakic B and Naumann K (2007) Arabidopsis histone deacetylase 6: a green link to RNA silencing. Oncogene 26, 5477-5488

31. Luo M, Cheng K, Xu Y, Yang S and Wu K (2017) Plant Responses to Abiotic Stress Regulated by Histone Deacetylases. Front Plant Sci 8, 2147

32. Ehrentraut S, Weber JM, Dybowski JN, Hoffmann D and Ehrenhofer-Murray AE (2010) Rpd3-dependent boundary formation at telomeres by removal of Sir2 substrate. Proc Natl Acad Sci U S A 107, 5522-5527

33. Zhou J, Zhou BO, Lenzmeier BA and Zhou JQ (2009) Histone deacetylase Rpd3 antagonizes Sir2-dependent 
silent chromatin propagation. Nucleic Acids Res 37, 3699-3713

34. Thurtle-Schmidt DM, Dodson AE and Rine J (2016) Histone Deacetylases with Antagonistic Roles in Saccharomyces cerevisiae Heterochromatin Formation. Genetics 204, 177-190

35. Suka N, Luo K and Grunstein M (2002) Sir2p and Sas2p opposingly regulate acetylation of yeast histone $\mathrm{H} 4$ lysine 16 and spreading of heterochromatin. Nat Genet 32, 378-383

36. Kimura A, Umehara $T$ and Horikoshi M (2002) Chromosomal gradient of histone acetylation established by Sas $2 p$ and Sir $2 p$ functions as a shield against gene silencing. Nat Genet 32, 370-377

37. Grafi G, Ben-Meir H, Avivi Y, Moshe M, Dahan Y and Zemach A (2007) Histone methylation controls telomerase-independent telomere lengthening in cells undergoing dedifferentiation. Dev Biol 306, 838-846

38. Vaquero-Sedas MI, Gámez-Arjona FM and Vega-Palas MA (2011) Arabidopsis thaliana telomeres exhibit euchromatic features. Nucleic Acids Res 39, 2007-2017

39. Zhou Y, Wang Y, Krause K et al (2018) Telobox motifs recruit CLF/SWN-PRC2 for H3K27me3 deposition via TRB factors in Arabidopsis. Nat Genet 50, 638-644

40. Colot V and Rossignol JL (1999) Eukaryotic DNA methylation as an evolutionary device. Bioessays 21, 402-411

41. Chan SW, Henderson IR and Jacobsen SE (2005) Gardening the genome: DNA methylation in Arabidopsis thaliana. Nat Rev Genet 6, 351-360

42. Cedar $\mathrm{H}$ and Bergman $\mathrm{Y}$ (2009) Linking DNA methylation and histone modification: patterns and paradigms. Nat Rev Genet 10, 295-304

43. Lindroth AM, Shultis D, Jasencakova Z et al (2004) Dual histone H3 methylation marks at lysines 9 and 27 required for interaction with CHROMOMETHYLASE3. EMBO J 23, 4146-4155

44. Liu X, Yu CW, Duan J et al (2012) HDA6 Directly Interacts with DNA Methyltransferase MET1 and Maintains Transposable Element Silencing in Arabidopsis. Plant Physiol 158, 119-129

45. To TK, Kim JM, Matsui A et al (2011) Arabidopsis HDA6 Regulates Locus-Directed Heterochromatin Silencing in Cooperation with MET1. PLoS Genet 7, e1002055

46. Vrbsky J, Akimcheva S and Watson JM (2010) siRNA-Mediated Methylation of Arabidopsis Telomeres. PLoS Genet 6, e1000986

47. Mathieu O, Probst AV and Paszkowski J (2005) Distinct regulation of histone $\mathrm{H} 3$ methylation at lysines 27 and 9 by $\mathrm{CpG}$ methylation in Arabidopsis. EMBO J 24, 2783-2791

48. Jacob Y, Feng S, LeBlanc CA et al (2009) ATXR5 and ATXR6 are H3K27 monomethyltransferases required for chromatin structure and gene silencing. Nat Struct Mol Biol 16, 763-768

49. Jacob Y, Stroud H, Leblanc C et al (2010) Regulation of heterochromatic DNA replication by histone $\mathrm{H} 3$ lysine 27 methyltransferases. Nature 466, 987-991
50. Raynaud C, Sozzani R, Glab N et al (2006) Two cell-cycle regulated SET-domain proteins interact with proliferating cell nuclear antigen (PCNA) in Arabidopsis. Plant J 47, 395-407

51. Brzeski J and Jerzmanowski A (2003) Deficient in DNA methylation 1 (DDM1) defines a novel family of chromatin-remodeling factors. J Biol Chem 278, 823-828

52. Zemach A, Kim MY, Hsieh PH et al (2013) The Arabidopsis nucleosome remodeler DDM1 allows DNA methyltransferases to access $\mathrm{H} 1$-containing heterochromatin. Cell 153, 193-205

53. Xie X and Shippen DE (2018) DDM1 guards against telomere truncation in Arabidopsis. Plant Cell Rep 37, 501-513

54. Richards EJ and Elgin SC (2002) Epigenetic codes for heterochromatin formation and silencing: Rounding up the usual suspects. Cell 108, 489-500

55. Fuchs J, Demidov D, Houben A and Schubert I (2006) Chromosomal histone modification patterns - from conservation to diversity. Trends Plant Sci 11, 199-208

56. Benetti R, Schoeftner S, Muñoz P and Blasco MA (2008) Role of TRF2 in the assembly of telomeric chromatin. Cell Cycle 7, 3461-3468

57. Benetti R, García-Cao M and Blasco MA (2007) Telomere length regulates the epigenetic status of mammalian telomeres and subtelomeres. Nat Genet 39, 243-250

58. Cubiles MD, Barroso S, Vaquero-Sedas MI, Enguix A, Aguilera A and Vega-Palas MA (2018) Epigenetic features of human telomeres. Nucleic Acids Res 46, 2347-2355

59. Conomos D, Stutz MD, Hills M et al (2012) Variant repeats are interspersed throughout the telomeres and recruit nuclear receptors in ALT cells. J Cell Biol 199, 893-906

60. O'Sullivan RJ and Almouzni G (2014) Assembly of telomeric chromatin to create ALTernative endings. Trends Cell Biol 24, 675-685

61. Vaquero-Sedas MI, Luo C and Vega-Palas MA (2012) Analysis of the epigenetic status of telomeres by using ChIP-seq data. Nucleic Acids Res 40, e163

62. Vaquero-Sedas MI and Vega-Palas MA (2013) Differential association of Arabidopsis telomeres and centromeres with histone $\mathrm{H} 3$ variants. Sci Rep 3, 1202

63. Vega-Vaquero A, Bonora G, Morselli M et al (2016) Novel features of telomere biology revealed by the absence of telomeric DNA methylation. Genome Res 26, 1047-1056

64. Galati A, Magdinier F, Colasanti V et al (2012) TRF2 controls telomeric nucleosome organization in a cell cycle phase-dependent manner. PLoS One 7, e34386

65. Deng Z, Norseen J, Wiedmer A, Riethman H and Lieberman PM (2009) TERRA RNA binding to TRF2 facilitates heterochromatin formation and ORC recruitment at telomeres. Mol Cell 35, 403-413

66. Lee WK, Yun JH, Lee $\mathrm{W}$ and Cho MH (2012) DNA-Binding Domain of AtTRB2 Reveals Unique Features of a Single Myb Histone Protein Family that Binds to Both Arabidopsis- and Human-Type Telomeric DNA Sequences. Mol Plant 5, 1406-1408 